\title{
Evolution of genetic and physiological mechanisms of cooperative behaviour
}

Michael Taborsky* and Barbara Taborsky

Behavioural Ecology, Institute of Ecology and Evolution, University of Bern

Address: Ethologische Station Hasli, Wohlenstrasse 50a, CH-3032 Hinterkappelen, Switzerland

* Corresponding author; michael.taborsky@iee.unibe.ch

Tel: +41316319156 


\begin{abstract}
Emergence of cooperative behaviour requires the evolution of proximate mechanisms to overcome the conflict of fitness interests inherent in social interactions. Reciprocal trading and enforcement of help are means by which cooperation can ensue between social partners subject to conflicting fitness interests. We discuss potential routes to the evolution of mechanisms of cooperative behaviour, with a special focus on vertebrates. We emphasize that a stable social organization, the exertion of social control and the experience-based propensity to act cooperatively are fundamental building blocks of cooperation. Genetic, neural and endocrine mechanisms involved in the regulation of cooperative behaviour are apparently deeply conserved in the phylogenies of different taxonomic groups. At the same time, the functions of regulatory systems diverge enormously between taxa, revealing functional independence and modular evolution of key components at both genetic and physiological levels.
\end{abstract}

\title{
The evolution of proximate mechanisms of behaviour
}

Evolution-oriented behavioural biology is currently experiencing a paradigm shift from a rather narrow focus on the functions and adaptive nature of traits to a more comprehensive approach incorporating psychological, physiological, genetic, ontogenetic and epigenetic mechanisms [1,2]. This reflects a recollection of the initial aims of ethology, which have been aptly summarized in Niko Tinbergen's seminal paper on that theme [3]. The recent urge for a more comprehensive understanding of behaviour, after a period in which 'behavioural ecology' with its tight slant on adaptive value has dominated the field, coincides with methodological developments now allowing researchers to ask questions about regulatory mechanisms at physiological and genetic levels, that is unprecedented [4,5,6**]. This broader approach to studying behaviour enables us, for the first time in the history of the biological study of behaviour, to address the pertinent question about the evolution of proximate mechanisms underlying behaviour $[2,7 * *]$.

\section{An integrative approach to the study of social behaviour}

Animals regularly interact with conspecifics, which can be both each other's strongest allies and fiercest competitors for resources. The resulting conflict and cooperation characterizing 
many intraspecific interactions selects for adequate responses to the behaviour of social partners [8**]. Like any other behavioural trait, these interactions are the products of genetic and physiological mechanisms that affect Darwinian fitness and evolve via natural selection [6**]. Comparative analyses suggest that in both social insects and vertebrates, regulatory elements of social behaviour at genetic and (neuro)physiological levels are remarkably conserved $\left[7^{* *}, 9,10^{* *}\right]$. Hence it seems obvious to ask whether and to which extent the evolution of cooperative behaviour, arguably the conundrum of Darwinian rationale, is influenced by the functionality and constraints of underlying genetic, epigenetic, endocrine, and neural mechanisms.

\section{Where do we find cooperation, and how can it evolve?}

Cooperation refers to the simultaneous or consecutive acting together of two or more individuals by same or different behaviours. It can evolve when all partners of a cooperative action obtain a fitness benefit from their behaviour, i.e. when the fitness benefits of the action outweigh its costs in the short or long term to each participant either by mutualism, reciprocity or correlated pay-offs (e.g., involving relatedness). Alternatively, the behaviour may be beneficial only to the receiver of a cooperative act, who can coerce another individual to behave cooperatively. In mutualistic interactions, there is typically no conflict of fitness interest between social partners, because the behaviour straightforwardly raises the direct fitness of the actor [11]. A familiar example of mutualistic interactions is group hunting, where individuals share forces to obtain prey, which typically provides a net benefit to all participants [12]. In case of mutualistic interactions the mechanisms of the involved behaviours can evolve by the same principles as any non-cooperative behaviour, because the potential fitness consequences to a social partner hardly matter. By the same token, if individuals act cooperatively towards others because their fitness pay-offs are correlated due to relatedness between them, i.e. because the behaviour is selected by indirect fitness benefits to the cooperator, there is usually little conflict of fitness interests. This applies for instance to many cooperatively breeding birds (e.g.[13]). More interesting for the question how the mechanisms controlling cooperative behaviour can evolve is the case where there is a distinct conflict of fitness interests between the involved parties. In that case, behavioural mechanisms will be selected that maximize the benefits of one party at the expense of another [14]. We shall further focus on this possibility. 
If there is a conflict of fitness interests between social partners, cooperation can evolve by reciprocity or coercion. Individuals may receive fitness returns by cooperation if their interaction partners pay back received help, either concurrently or in the future. Reciprocal cooperation or 'trading' of that kind usually involves negotiation between social partners [15], which involves rules that are subject to selection [16,17]. Reciprocity and trading require a number of cognitive abilities, including individual (or 'class') recognition, the ability to relate the behaviour of others to own pay-offs, and a certain degree of social memory. The requirement for social memory depends on whether actions and reactions occur concurrently ('coaction') or with some time lag ('reciprocity'[18*]). Reciprocity and trading has been demonstrated in a wide range of vertebrates, from fish to mammals [19-22]. Social bonds can favour such exchanges of cooperative behaviours [23]. Both the neuroendocrine 'social behaviour network' involving steroid and neuropeptide hormones, and the mesolimbic reward system involving dopaminergic signalling pathways constitute important regulatory mechanisms responsible for the concerned behaviours $[4,7 * *]$.

A much simpler form of reciprocal cooperation can ensue if individuals 'return' received help to others, i.e. not to the individual from which they received help. Such 'generalized reciprocity' has been shown to afford evolutionarily stable levels of cooperation under a wide range of conditions (e.g. [24,25]). This cooperation mechanism is particularly intriguing because it does not require specific cognitive abilities, except the recognition of received help [26,27]. Hitherto it has been experimentally demonstrated in rats, monkeys and humans, but because of its mechanistic simplicity, it is expected to be much more widespread $[26,28,29]$. In vertebrates, neuroendocrine regulation of generalized reciprocity may be mediated primarily by the nonapeptide system, particularly oxytocin or its orthologs [30-32].

One social partner forcing another one to cooperate constitutes the second possibility to generate cooperative behaviour among social partners subject to a conflict of fitness interests. Enforced cooperation can be evolutionarily stable if the costs to avoid such manipulation are higher for an individual than to accept being exploited. The generation of cooperation by this means requires some sort of dominance asymmetry between social partners, the ability of the receiver of enforcement to respond appropriately to the dominant's demand, and the ability of the dominant to accept the resulting cooperation as consummatory response. Enforced cooperation occurs in a wide range of taxa and contexts [33-38]. Steroid hormones are 
important regulatory components of social interactions based on dominance asymmetries, which can affect the cooperation propensity of subordinates [39-41].

Hitherto, cooperation between social partners with a conflict of fitness interests has been studied mainly regarding its mode of action and its potential adaptive value, without considering underlying psychological, physiological and genetic mechanisms. If we wish to fully understand the evolution of cooperation, however, we need to unravel the required components, the involved decision rules, the ecological functionality, potential synergistic effects and eco-evolutionary feedbacks, trait evolvability and mechanistic constraints $[6,42,43,44 *, 45-49,50 *, 51]$. In particular, we need to understand the emergence of novel traits that are prerequisites for such seemingly demanding interaction, and the mechanistic building blocks of cooperative behaviour, taking into account the effect of potential conflict of fitness interests on the evolution of involved proximate mechanisms.

\section{Routes to the evolution of mechanisms of cooperative behaviour}

Obviously, the evolution of sociality from solitary ancestry and the evolution of cooperative from non-cooperative behaviours require the emergence of novel social traits, such as individual or 'class' recognition and social memory. High degrees of pleiotropy would naturally limit the emergence of novel traits, but recent analyses quantifying the degree of pleiotropy across different organisms revealed that it is much more restricted than previously thought, and that genotype-phenotype maps are highly modular [52].

Two main evolutionary routes to novelty in social traits have been evaluated in cooperative animal societies, hitherto best exemplified in social insects. (1) Evolving new social behaviours from ancestral gene networks that gain increased regulatory flexibility and are coopted for a new function; (2) emergence of genetic novelty, which is particuarly required for the evolution of the most advanced forms of eusociality [53-55]. The first route builds on the existence of distinct, ancestral molecular modules regulating the physiology and behaviour of solitary species, which have undergone modification during the transition to (eu)sociality. These distinct modules are combined to produce social pathways orchestrating all steps of an appropriate behavioural expression from perception of a stimulus to the final motor action. Regulatory processes are connected strongly within, but only weakly between modules, allowing largely independent module evolution and functional flexibility [54]. An example 
for co-opting of conserved elements in this context is the gene network around the juvenile hormone $(\mathrm{JH})$ and vitellogenin $(\mathrm{Vg})$. In solitary insects and reproductive queens, $\mathrm{JH}$ upregulates $\mathrm{Vg}$ and stimulates oocyte maturation, while in workers of eusocial bees, ants and termites, the two genes inhibit each other [56].

Evolution of novel genes, the second major route to evolutionary novelty, is assumed to be associated with later stages in the transition from solitary to (eu)social life style [10**]. It can be identified with help of 'taxonomically restricted genes' (TGRs). In the honey bee genome, 700 such TGRs were detected, which exist solely either in the honey bee, in hymenoptera, or in insects [55]. These genes were found to underlie disproportionately often novel phenotypes involving worker traits, and to undergo rapid changes of coding sequence [57]. Remarkably, independent evolutionary transitions from solitary to (eu)social life styles differ in their particular genomic changes, but the different transitions have similar general features [58].

Here we aim to evaluate whether the same two general routes to evolve cooperative behaviours, namely co-opting of conserved elements and de novo evolution, may likewise apply in vertebrates. While molecular mechanisms generating genetic novelty are not yet well explored in vertebrates, several conserved signalling systems are known to be involved in the behavioural and physiological building blocks of vertebrate cooperation. Here we identify three building blocks and discuss their underlying regulatory mechanisms, and the insights thus far achieved regarding their evolution.

\section{Proximate building blocks of cooperation}

The first building block of cooperation is a stable social organization that remains functional even if conflicts of interest occur among individuals. Unnecessary friction or repeated negotiations of dominance relationships and task commitment can be avoided if individuals express appropriate social behaviours dependent on their (relative) social state and the particular context $\left[8^{* *}\right]$. Developmental processes and current social stimuli underlie the expression of appropriate social behaviour. A key developmental mechanism in this context is the early acquisition of social competence, i.e. the ability of individuals to flexibly use social information in order to optimize their social performance [8**]. The early programming of the vertebrate stress axis [hypothalamic-pituitary-adrenal (HPA) or -interrenal (HPI) axis] seems central for determining social performance later in life: in fish, mice and rats, social enrichment results in both (i) a lower stress responsiveness, as revealed by the expression of 
stress axis genes in the brain and by behavioural test results, and (ii) a better performance during social challenges $\left[59,60,61^{*}\right]$. In the cooperatively breeding cichlid $N$. pulcher, for instance, these behavioural effects enhance the functionality of social groups by increasing the tolerance of subordinates and reducing the duration of contests $[62,63]$. Furthermore, in rodents the development of social competence is accompanied by higher concentrations of the neural plasticity marker BDNF in the brain [64].

Immediate social experience may also trigger the physiological state of an animal in order to prepare it to perform appropriate social behaviour. For example, watching a fight between conspecifics changes the physiological state of a bystander in the cichlid Oreochromis mossambicus [65]: the bystander experiences a surge of androgens, probably thereby getting prepared for an aggressive encounter in the near future. Hormonal systems are known to generally affect sociability, which is a precondition for group life. The oxytocin-like peptides including oxytocin (mammals), mesotocin (birds, reptiles and amphibians) and isotocin (fish), and their receptors, which form the vertebrate nonapeptide signalling system together with arginine vasotocin/vasopressin, have often been highlighted as the promotor of social behaviour. However, due to the modular nature of the nonapeptide system and its speciesspecific evolution [66*], general predictions of the direction of effects can often not be made, even within a vertebrates class. For instance, while in humans, non-human mammals and goldfish oxytocin/isotocin favours social approach [66*], in the cooperatively breeding cichlid $N$. pulcher it has exactly the opposite effect [67], while no effect was observed on approach behaviour in zebra finches [66*]. In general, long-term social relationships such as pair or family bonds may help to reduce conflict. However, the physiological regulation of pair bonding involving the vasopressin or the oxytocin system seems to strongly diverge between different vertebrate taxa $\left[66^{*}\right]$, revealing that taxon matters when studying mechanisms of social behaviour [68]. In fact, to date there is no clear evidence that nonapeptides are at all causally involved in the process of bonding.

The second building block of cooperation between individuals that are subject to a conflict of fitness interests is the exertion of social control to prevent cheating, and to manifest the division of tasks between dominants and subordinates. Here, differences in social rank play an important role, which are often associated with differential sex steroid profiles, in particular variation in androgen and progesterone levels (cichlids: [39,69] mole rats: [70]). High rank often needs to be persistently reinforced, which typically occurs by dominants' aggression. 
Also here, the functionality of the underlying hormonal regulation may diverge between species. For instance, activation of the AVT system has been assumed to favour aggressive tendencies in dominants of mouthbrooding cichlids [71], but in the cooperatively breeding cichlid $N$. pulcher no association between aggression and AVT could be identified [72]. Furthermore, comparative evidence suggests that an induction of increased stress levels in subordinates by repeated aggression of dominants is unlikely to act as general control mechanism in cooperative breeders, as dominants often have higher corticoid levels than subordinates [73]. However, during specific critical circumstances, such as during a dominant female's pregnancy (meercats [74]), or because of particularly harsh environmental conditions (superb starlings [75]), dominant aggression can effectively restrain subordinate reproduction through an activation of the HPA axis, with downstream effects on gonadotropin-releasing hormone in the hypothalamus and resultant blocking of ovulation. A similar pathway was proposed to be involved in species in which dominants can achieve complete reproductive suppression of subordinates, such as in eusocial naked mole rats and Damaraland mole rats [76].

Alternatively to active control by dominant aggression, subordinates can pre-emptively provide honest signals of submission or of their helping propensity [77], or of low reproductive intent [39]. For example, the circulating levels of androgens are lower in male helpers of $N$. pulcher showing high levels of submission towards breeder males, thereby conveying an honest signal of their low reproductive potential [39]. Subordinates can exhibit self-imposed reproductive suppression in systems where dominants would inevitably kill subordinates' offspring. In common marmosets, the pituitary gonadotropin levels dropped rapidly and ovulation stopped when individuals were introduced into groups where they had to obtain a subordinate role [78].

Mechanisms of the third building block of cooperation, the actual propensity to behave cooperatively, have been predominantly studied in vertebrates in the context of reciprocity or when commodities are exchanged, with a focus on the role of the nonapeptide system. Like for the effect of nonapeptides on social approach, general trends appear to be absent. Activation of the oxytocin (humans: [79]) and the AVT/AVP system (humans: [80]) were found to enhance cooperative tendencies as often as they were reported to inhibit cooperation (OT: humans [81]; AVT: cleaner wrasse [82]) or to exert effects strongly contingent on social context (OT: humans $[80,83])$. Experimental work on Norway rats suggests that the effects of 
oxytocin on reciprocal cooperation may be mediated mainly by its role in modulating social anxiety rather than a specific effect on the generation of trust (M. Dietz \& M. Taborsky, unpubl.). A general anxiolytic effect of oxytocin seems to affect cooperation propensity also in capuchin monkeys [84].

Notably, the physiological mechanisms mediating helping behaviour in cooperative breeders are hitherto not well understood. Thus far the role of two hormones has been studied more closely in meerkats, cortisol and prolactin. Their effects seem to depend on the particular care behaviour considered. Cortisol but not prolactin was positively associated with alloparental pup-feeding [85]. In contrast higher prolactin but lower cortisol levels immediately advanced babysitting in male helpers of this species [86].

\section{Conclusions}

When compiling existing information about the mechanisms underlying cooperative behaviour in vertebrates and its essential building blocks embedded in social interactions, at first glance the picture seems blurred. It becomes quickly clear, however, that the same regulatory systems often have divergent functions and effects in different taxa. Despite the obvious conservation of neural and neuroendocrine components among very divergent vertebrate lineages $\left[7^{* *}, 66^{*}\right]$, the functions of the involved regulatory systems seem to vary substantially, suggesting independence by modular evolution. Two candidates stand out as being involved apparently in all three building blocks of cooperation, the vertebrate stress axis and the nonapeptide system. They act in very different ways, though, often showing directly opposing effects in different species. To some extent this resembles the regulatory shifts in 'old' genes to new functions in the social insects [10**]. The enormous variety of cooperative behaviours shown in vertebrates and the flexibility of their context-dependent expression are unlikely to be governed merely by two hormonal systems, however. We are only just beginning to understand the regulatory and signalling networks responsible for the expression of vertebrate cooperation.

\section{Acknowledgements}

Research leading to this article was supported by the Swiss National Science Foundation (grants 31003A_156152 to MT and 31003A_156881 to BT) 


\section{Reference List}

1. Taborsky M. (2014). Tribute to Tinbergen: The Four Problems of Biology. A Critical Appraisal. Ethology 120: 224-227.

2. Fawcett T.W., Marshall J.A., and Higginson A.D. (2015). The Evolution of Mechanisms Underlying Behaviour. Current Zoology 61: 221-225.

3. Tinbergen N. (1963). On aims and methods of Ethology. Zeitschrift für Tierpsychologie 20: 410-433.

4. O'Connell L.A. and Hofmann H.A. (2011). The Vertebrate mesolimbic reward system and social behavior network: A comparative synthesis. Journal of Comparative Neurology 519: 3599-3639.

5. Springer S.A., Crespi B.J., and Swanson W.J. (2011). Beyond the phenotypic gambit: molecular behavioural ecology and the evolution of genetic architecture. Molecular Ecology 20: 2240-2257.

6. Hofmann H.A., Beery A.K., Blumstein D.T., Couzin I.D., Earley R.L., Hayes L.D., Hurd P.L., Lacey E.A., Phelps S.M., Solomon N.G. et al. (2014). An evolutionary framework for studying mechanisms of social behavior. Trends in Ecology \& Evolution 29: 581-589.**

This paper outlines a comprehensive evolutionary approach combining internal attributes (neural, molecular and life-history traits), external attributes (ecological and social parameters) and the functional context of social grouping (incorporating the functions of social behaviors) in the study of mechanisms underlying social behavior and cooperation.

7. O'Connell L.A. and Hofmann H.A. (2012). Evolution of a Vertebrate Social DecisionMaking Network. Science 336: 1154-1157.**

The comparative analysis presented in this study suggests that brain regions involved in behavioural regulation have been deeply conserved over 450 million years of vertebrate evolution. However, different brain regions have apparently experienced different selection pressures, thus allowing for the evolution of a striking diversity of social and cooperative behaviours in vertebrates.

8. Taborsky B. and Oliveira R.F. (2012). Social competence: an evolutionary approach. Trends in Ecology \& Evolution 27: 679-688.**

This conceptual article proposes an evolutionary approach to the study of social competence that is, the ability of individuals to optimize the outcome of social interactions by adjusting their behaviour to specific social information. Optimal behavioural responses to the social environment are discussed regarding their adaptive value, involved costs, underlying proximate mechanisms, and potential trade-offs.

9. Simola D.F., Wissler L., Donahue G., Waterhouse R.M., Helmkampf M., Roux J., Nygaard S., Glastad K.M., Hagen D.E., Viljakainen L. et al. (2013). Social insect genomes exhibit dramatic evolution in gene composition and regulation while preserving regulatory features linked to sociality. Genome Research 23: 1235-1247.

10. Rehan S.M. and Toth A.L. (2015). Climbing the social ladder: the molecular evolution of sociality. Trends in Ecology \& Evolution 30: 426-433.**

This review summarizes several hypotheses aiming to explain the molecular evolution of eusociality in insects. It provides a synthetic framework proposing that different evolutionary processes have dominated during different transitional stages of social evolution, ranging from adaptive changes in gene regulation early in this trajectory to the emergence of novel genes later on. 
11. Lehmann L. and Keller L. (2006). The evolution of cooperation and altruism - a general framework and a classification of models. Journal of Evolutionary Biology 19: 1365-1376.

12. Packer C. and Ruttan L. (1988). The Evolution of Cooperative Hunting. American Naturalist 132: 159-198.

13. Hatchwell B.J., Gullett P.R., and Adams M.J. (2014). Helping in cooperatively breeding long-tailed tits: a test of Hamilton's rule. Philosophical Transactions of the Royal Society B-Biological Sciences 369.

14. West S.A., Griffin A.S., and Gardner A. (2007). Evolutionary explanations for cooperation. Current Biology 17: R661-R672.

15. Taylor P.D. and Day T. (2004). Stability in negotiation games and the emergence of cooperation. Proceedings of the Royal Society B-Biological Sciences 271: 669-674.

16. McNamara J.M., Gasson C.E., and Houston A.I. (1999). Incorporating rules for responding into evolutionary games. Nature 401: 368-371.

17. McNamara J.M. and Houston A.I. (2009). Integrating function and mechanism. Trends in Ecology \& Evolution 24: 670-675.

18. Van Doorn G., Riebli T., and Taborsky M. (2014). Coaction versus reciprocity in continuous-time models of cooperation. Journal of Theoretical Biology 356: 1-10.*

This game theory model reveals that introducing a time delay between the mutual actions and reactions of cooperating partners has effects for the emergence and evolutionary stability of cooperation that are similar to increasing the costs of cooperation. Thereby, this model adds important realism to the study of cooperation by mutual exchange, showing that cooperative coaction (concurrent exchange of help and services) can evolve much easier than reciprocal cooperation (characterized by delayed response opportunities).

19. Seyfarth R.M. and Cheney D.L. (1984). Grooming, Alliances and Reciprocal Altruism in Vervet Monkeys. Nature 308: 541-543.

20. Rutte C. and Taborsky M. (2008). The influence of social experience on cooperative behaviour of rats (Rattus norvegicus): direct vs generalised reciprocity. Behavioral Ecology and Sociobiology 62: 499-505.

21. Carter G.G. and Wilkinson G.S. (2013). Food sharing in vampire bats: reciprocal help predicts donations more than relatedness or harassment. Proceedings of the Royal Society B-Biological Sciences 280.

22. Zoettl M., Heg D., Chervet N., and Taborsky M. (2013). Kinship reduces alloparental care in cooperative cichlids where helpers pay-to-stay. Nature Communications 4.

23. St-Pierre A., Larose K., and Dubois F. (2009). Long-term social bonds promote cooperation in the iterated Prisoner's Dilemma. Proceedings of the Royal Society BBiological Sciences 276: 4223-4228.

24. Rankin D.J. and Taborsky M. (2009). Assortment and the Evolution of Generalized Reciprocity. Evolution 63: 1913-1922.

25. van Doorn G.S. and Taborsky M. (2012). The Evolution of Generalized Reciprocity on Social Interaction Networks. Evolution 66: 651-664.

26. Rutte C. and Taborsky M. (2007). Generalized reciprocity in rats. Plos Biology 5: 1421-1425.

27. Barta Z., McNamara J.M., Huszar D.B., and Taborsky M. (2011). Cooperation among non-relatives evolves by state-dependent generalized reciprocity. Proceedings of the Royal Society B-Biological Sciences 278: 843-848.

28. Bartlett M.Y. and DeSteno D. (2006). Gratitude and prosocial behavior. Psychological Science 17: 319-325.

29. Leimgruber K.L., Ward A.F., Widness J., Norton M.I., Olson K.R., Gray K., and Santos L.R. (2014). Give What You Get: Capuchin Monkeys (Cebus apella) and 4- 
Year-Old Children Pay Forward Positive and Negative Outcomes to Conspecifics. Plos One 9.

30. Barraza J. and Zak P.J. (2009). Empathy toward Strangers Triggers Oxytocin Release and Subsequent Generosity.).

31. Barraza J.A., McCullough M.E., Ahmadi S., and Zak P.J. (2011). Oxytocin infusion increases charitable donations regardless of monetary resources. Hormones and Behavior 60: 148-151.

32. Klackl J., Pfundmair M., Agroskin D., and Jonas E. (2013). Who is to blame? Oxytocin promotes nonpersonalistic attributions in response to a trust betrayal. Biological Psychology 92: 387-394.

33. Reeve H.K. and Gamboa G.J. (1987). Queen Regulation of Worker Foraging in Paper Wasps - A Social Feedback-Control System (Polistes Fuscatus, Hymenoptera, Vespidae). Behaviour 102: 147-167.

34. Reeve H.K. (1992). Queen Activation of Lazy Workers in Colonies of the Eusocial Naked Mole-Rat. Nature 358: 147-149.

35. Clutton-Brock T.H. and Parker G.A. (1995). Punishment in animal societies. Nature 373: 209-216.

36. Tebbich S., Taborsky M., and Winkler H. (1996). Social manipulation causes cooperation in keas. Animal Behaviour 52: 1-10.

37. Fehr E. and Gachter S. (2002). Altruistic punishment in humans. Nature 415: 137-140.

38. Fischer S., Zottl M., Groenewoud F., and Taborsky B. (2014). Group-size-dependent punishment of idle subordinates in a cooperative breeder where helpers pay to stay. Proceedings. Biological sciences / The Royal Society 281.

39. Bender N., Heg D., Hamilton I.M., Bachar Z., Taborsky M., and Oliveira R.F. (2006). The relationship between social status, behaviour, growth and steroids in male helpers and breeders of a cooperatively breeding cichlid. Hormones and Behavior 50: 173182.

40. Bender N., Heg-Bachar Z., Oliveira R.F., Canario A.V., and Taborsky M. (2008). Hormonal control of brood care and social status in a cichlid fish with brood care helpers. Physiology \& Behavior 94: 349-358.

41. Brouwer L., Groothuis T.G., Vedder O., Eikenaar C., Richardson D.S., and Komdeur J. (2009). Do Primary Males Physiologically Suppress Subordinate Males? An Experiment in a Cooperatively Breeding Passerine. Ethology 115: 576-587.

42. Akcay E., Van Cleve J., Feldman M.W., and Roughgarden J. (2009). A theory for the evolution of other-regard integrating proximate and ultimate perspectives. Proceedings of the National Academy of Sciences of the United States of America 106: 1906119066.

43. Katz P.S. (2011). Neural mechanisms underlying the evolvability of behaviour. Philosophical Transactions of the Royal Society B-Biological Sciences 366: 20862099.

44. Fawcett T.W., Fallenstein B., Higginson A.D., Houston A.I., Mallpress D.E., Trimmer P.C., and McNamara J.M. (2014). The evolution of decision rules in complex environments. Trends in Cognitive Sciences 18: 153-161.*

This article aims at explaining biases in decision making within an evolutionary framework. The authors argue that typically the real world is complex, variable and autocorrelated, which should select for a significant response of perceptual and cognitive systems to the statistical structure of real-world environments. This logic is particularly important regarding decisions involving social behaviour and cooperation.

45. Fawcett T.W., Hamblin S., and Giraldeau L.A. (2013). Exposing the behavioral gambit: the evolution of learning and decision rules. Behavioral Ecology 24: 2-11. 
46. Giske J., Eliassen S., Fiksen O., Jakobsen P.J., Aksnes D.L., Jorgensen C., and Mangel M. (2013). Effects of the Emotion System on Adaptive Behavior. American Naturalist 182: 689-703.

47. Giske J., Eliassen S., Fiksen O., Jakobsen P.J., Aksnes D.L., Mangel M., and Jorgensen C. (2014). The emotion system promotes diversity and evolvability. Proceedings of the Royal Society B-Biological Sciences 281.

48. McNamara J.M. (2013). Towards a richer evolutionary game theory. Journal of the Royal Society Interface 10.

49. Trimmer P.C. and Houston A.I. (2014). An Evolutionary Perspective on Information Processing. Topics in Cognitive Science 6: 312-330.

50. van den Berg P. and Weissing F.J. (2015). The importance of mechanisms for the evolution of cooperation. Proceedings. Biological sciences / The Royal Society 282.*

The simulation model discussed here assumes four different behavioural strategies based on differences in behavioural architecture and mutation regimes. Assuming two different pay-off matrices, the results suggest that the probability that specific behavioural strategies arise depends on the underlying behavioural and genetic architectures, which has strong implications for the evolutionary outcome.

51. van der Post D.J., Verbrugge R., and Hemelrijk C.K. (2015). The Evolution of Different Forms of Sociality: Behavioral Mechanisms and Eco-Evolutionary Feedback. Plos One 10.

52. Wagner G.P. and Zhang J. (2011). The pleiotropic structure of the genotypephenotype map: the evolvability of complex organisms. Nature Reviews Genetics 12: 204-213.

53. Johnson B.R. and Linksvayer T.A. (2010). Deconstructing the Superorganism: Social Physiology, Groundplans, and Sociogenomics. Quarterly Review of Biology 85: 5779.

54. Bloch G. and Grozinger C.M. (2011). Social molecular pathways and the evolution of bee societies. Philosophical Transactions of the Royal Society B-Biological Sciences 366: 2155-2170.

55. Johnson B.R. and Tsutsui N.D. (2011). Taxonomically restricted genes are associated with the evolution of sociality in the honey bee. Bmc Genomics 12.

56. Amsalem E., Malka O., Grozinger C., and Hefetz A. (2014). Exploring the role of juvenile hormone and vitellogenin in reproduction and social behavior in bumble bees. Bmc Evolutionary Biology 14.

57. Jasper W.C., Linksvayer T.A., Atallah J., Friedman D., Chiu J.C., and Johnson B.R. (2015). Large-Scale Coding Sequence Change Underlies the Evolution of Postdevelopmental Novelty in Honey Bees. Molecular Biology and Evolution 32: 334-346.

58. Kapheim K.M., Pan H., Li C., Salzberg S.L., Puiu D., Magoc T., Robertson H.M., Hudson M.E., Venkat A., Fischman B.J. et al. (2015). Genomic signatures of evolutionary transitions from solitary to group living. Science 348: 1139-1143.

59. Curley J.P., Davidson S., Bateson P., and Champagne F.A. (2009). Social enrichment during postnatal development induces transgenerational effects on emotional and reproductive behavior in mice. Frontiers in Behavioral Neuroscience 3.

60. Branchi I., Santarelli S., D'Andrea I., and Alleva E. (2013). Not all stressors are equal: Early social enrichment favors resilience to social but not physical stress in male mice. Hormones and Behavior 63: 503-509.

61. Taborsky B., Tschirren L., Meunier C., and Aubin-Horth N. (2013). Stable reprogramming of brain transcription profiles by the early social environment in a cooperatively breeding fish. Proceedings of the Royal Society B-Biological Sciences 280: 20122605.* 
This paper shows that, like in mammals, also in ectotherm vertebrates such as fish the early programming of the stress axis is of central importance for the development of social competence, suggesting that this mechanism is deeply conserved within vertebrates. Moreover, experimental results provide evidence that the early programming of the stress axis entails effects on social decisions of individuals that remain stable over a lifetime.

62. Arnold C. and Taborsky B. (2010). Social experience in early ontogeny has lasting effects on social skills in cooperatively breeding cichlids. Animal Behaviour 79: 621630.

63. Taborsky B., Arnold C., Junker J., and Tschopp A. (2012). The early social environment affects social competence in a cooperative breeder. Animal Behaviour 83: 1067-1074.

64. Branchi I., Curley J.P., D'Andrea I., Cirulli F., Champagne F.A., and Alleva E. (2013). Early interactions with mother and peers independently build adult social skills and shape BDNF and oxytocin receptor brain levels. Psychoneuroendocrinology 38: 522532.

65. Oliveira R.F., Lopes M., Carneiro L.A., and Canario A.V.M. (2001). Watching fights raises fish hormone levels - Cichlid fish wrestling for dominance induce an androgen surge in male spectators. Nature 409: 475.

66. Goodson J.L. (2013). Deconstructing sociality, social evolution and relevant nonapeptide functions. Psychoneuroendocrinology 38: 465-478.*

This conceptual review discusses the functional evolution of the multi-variant regulatory effects of the vertebrate nonapeptide system (oxytocin, vasopressin and their orthologs), which is partly responsible for bonding, parental care, prosocial and antisocial behaviours in a wide range of vertebrates.

67. Reddon A.R., Voisin M.R., O'Connor C.M., and Balshine S. (2014). Isotocin and sociality in the cooperatively breeding cichlid fish, Neolamprologus pulcher. Behaviour 151: 1389-1411.

68. Taborsky M., Hofmann H.A., Beery A.K., Blumstein D.T., Hayes L.D., Lacey E.A., Martins E.P., Phelps S.M., Solomon N.G., and Rubenstein D.R. (2015). Taxon matters: promoting integrative studies of social behavior NESCent Working Group on Integrative Models of Vertebrate Sociality: Evolution, Mechanisms, and Emergent Properties. Trends in Neurosciences 38: 189-191.

69. Almeida O., Goncalves-de-Freitas E., Lopes J.S., and Oliveira R.F. (2014). Social instability promotes hormone-behavior associated patterns in a cichlid fish. Hormones and Behavior 66: 369-382.

70. Zhou S., Holmes M.M., Forger N.G., Goldman B.D., Lovern M.B., Caraty A., Kallo I., Faulkes C.G., and Coen C.W. (2013). Socially regulated reproductive development: Analysis of GnRH-1 and kisspeptin neuronal systems in cooperatively breeding naked mole-rats (Heterocephalus glaber). Journal of Comparative Neurology 521: 30033029.

71. Greenwood A.K., Wark A.R., Fernald R.D., and Hofmann H.A. (2008). Expression of arginine vasotocin in distinct preoptic regions is associated with dominant and subordinate behaviour in an African cichlid fish. Proceedings of the Royal Society BBiological Sciences 275: 2393-2402.

72. Reddon A.R., O'Connor C.M., Marsh-Rollo S.E., Balshine S., Gozdowska M., and Kulczykowska E. (2015). Brain nonapeptide levels are related to social status and affiliative behaviour in a cooperatively breeding cichlid fish. Royal Society open science 2: 140072.

73. Creel S. (2001). Social dominance and stress hormones. Trends in Ecology \& Evolution 16: 491-497. 
74. Young A.J., Carlson A.A., Monfort S.L., Russell A.F., Bennett N.C., and CluttonBrock T. (2006). Stress and the suppression of subordinate reproduction in cooperatively breeding meerkats. Proceedings of the National Academy of Sciences of the United States of America 103: 12005-12010.

75. Rubenstein D.R. (2007). Stress hormones and sociality: integrating social and environmental stressors. Proceedings of the Royal Society B-Biological Sciences 274: 967-975.

76. Voigt C., Gahr M., Leitner S., Lutermann H., and Bennett N. (2014). Breeding status and social environment differentially affect the expression of sex steroid receptor and aromatase mRNA in the brain of female Damaraland mole-rats. Frontiers in Zoology 11 .

77. Bergmüller R. and Taborsky M. (2005). Experimental manipulation of helping in a cooperative breeder: helpers 'pay to stay' by pre-emptive appeasement. Animal Behaviour 69: 19-28.

78. Saltzman W., Digby L.J., and Abbott D.H. (2009). Reproductive skew in female common marmosets: what can proximate mechanisms tell us about ultimate causes? Proceedings of the Royal Society B-Biological Sciences 276: 389-399.

79. Kosfeld M., Heinrichs M., Zak P.J., Fischbacher U., and Fehr E. (2005). Oxytocin increases trust in humans. Nature 435: 673-676.

80. Rilling J.K., DeMarco A.C., Hackett P.D., Thompson R., Ditzen B., Patel R., and Pagnoni G. (2012). Effects of intranasal oxytocin and vasopressin on cooperative behavior and associated brain activity in men. Psychoneuroendocrinology 37: 447461.

81. Bartz J., Simeon D., Hamilton H., Kim S., Crystal S., Braun A., Vicens V., and Hollander E. (2011). Oxytocin can hinder trust and cooperation in borderline personality disorder. Social Cognitive and Affective Neuroscience 6: 556-563.

82. Cardoso S., Paitioa J., Oliveira R.F., Bshary R., and Soares M. (2015). Arginine vasotocin reduces levels of cooperative behaviour in a cleaner fish. Physiology \& Behavior 139: 314-320.

83. De Dreu C.K. (2012). Oxytocin modulates cooperation within and competition between groups: An integrative review and research agenda. Hormones and Behavior 61: 419-428.

84. Brosnan S.F., Talbot C.F., Essler J.L., Leverett K., Flemming T., Dougall P., Heyler C., and Zak P.J. (2015). Oxytocin reduces food sharing in capuchin monkeys by modulating social distance. Behaviour 152: 941-961.

85. Carlson A.A., Manser M.B., Young A.J., Russell A.F., Jordan N.R., McNeilly A.S., and Clutton-Brock T. (2006). Cortisol levels are positively associated with pupfeeding rates in male meerkats. Proceedings of the Royal Society B-Biological Sciences 273: 571-577.

86. Carlson A.A., Russell A.F., Young A.J., Jordan N.R., McNeilly A.S., Parlow A.F., and Clutton-Brock T. (2006). Elevated prolactin levels immediately precede decisions to babysit by male meerkat helpers. Hormones and Behavior 50: 94-100. 